Recibido: 18/09/2020 --- Aceptado: 06/11/2020 --- Publicado: 12/04/2021

\title{
EDUCACIÓN PERFORMATIVA MUSICAL: HERRAMIENTAS PARA LA INVESTIGACIÓN
}

\section{MUSICAL PERFORMATIVE EDUCATION: RESEARCH TOOLS}

(8) Ana María Botella Nicolás ${ }^{1}$. Universitat de València. España. ana.maria.botella@uv.es

Guillem Escorihuela Carbonell ${ }^{2}$. Institut Superior d'Ensenyances Artístiques (ISEACV). España.

escorihuela gui@gva.es

\section{Cómo citar el artículo:}

Botella, A. M. y Escorihuela, G. (2021). Educación performativa musical: herramientas para la investigación. Revista de Comunicación de la SEECI, 54, 1-19. http://doi.org/10.15198/seeci.2021.54.e614

\section{RESUMEN}

Este artículo pretende dotar de herramientas al docente para que aborde la realidad de la clase práctica instrumental. A través de la metodología de complementación cuantitativa y cualitativa, se observa como ésta resulta la solución más indicada para este tipo de investigaciones. A partir de una reflexión sobre la clase musical instrumental y la investigación en didáctica de los instrumentos, el cuestionario se revela como la herramienta más completa para conocer la actitud del profesorado acerca de la praxis docente. Repasando algunas investigaciones sobre educación performativa se aportan ejemplos que permitan a los profesores enfrentarse a los retos que supone la educación musical en el siglo XXI. Por tanto, la metodología se basa en una revisión bibliográfica de fuentes primarias y secundarias sobre lo que han escrito los autores más influyentes en el campo de la educación performativa. Esta publicación quiere enfatizar en la importancia de la investigación a nivel educativo en el ámbito de la música, especialmente en la enseñanza instrumental, que tan falta de reflexión está en la actualidad. Por tanto, lo original del texto se

\footnotetext{
1 Ana María Botella Nicolás: Profesora contratada doctora del Departamento de Didáctica de la Expresión musical, Plástica y Corporal de la Facultad de Magisterio de la Universitat de València. ana.maria.botella@uv.es

2 Guillem Escorihuela Carbonell: Doctor en didáctica de la música por la Universitat de València. Es titulado Superior en Música en la especialidad de Flauta Travesera por el Conservatorio Superior de València. escorihuela gui@gva.es
} 
encuentra en los ejemplos que se presentan como modelos para el profesorado que quiera implementar una búsqueda en su propia docencia.

PALABRAS CLAVE: educación musical performativa - conservatorio - didáctica de la música - investigación educativa - Enseñanza musical - clase instrumental profesorado.

\section{ABSTRACT}

This article aims to provide the teacher with tools to address the reality of the instrumental practice class. Through the methodology of quantitative and qualitative complementation, it is appreciated how this is the most indicated solution for this type of research. From a reflection on the instrumental musical class and the research in didactic of the instruments, the questionnaire is revealed as the most complete tool to know the attitude of the teaching staff about the teaching praxis. Reviewing some research on performative education provides examples that allow teachers to face the challenges of music education in the 21st century. Therefore, the methodology is based on a literature review of primary and secondary sources on what the most influential authors have written in the field of performative education. This publication wants to emphasize the importance of educational research in the field of music, especially in instrumental teaching, which is so lacking in reflection today. Therefore, the original of the text is found in the examples that are presented as models for teachers who want to implement a search in their own teaching.

KEY WORDS: performative music education - conservatoire - didactic music educational research - teaching music - instrument class- teachers.

\section{EDUCAÇÃO PERFORMATIVA MUSICAL: FERRAMENTAS PARA A PESQUISA}

\section{RESUMO:}

Este artigo pretende fornecer ferramentas ao professor para a realidade das aulas práticas instrumentais. Através da metodologia de complementação quantitativa e qualitativa, se observa como a mesma resulta a solução mais indicada para este tipo de pesquisa. A partir de uma reflexão sobre a aula de música instrumental e a pesquisa na didática dos instrumentos, o questionário se revela como a ferramenta mais completa para conhecer a atitude dos professores sobre a prática do professor. Revisitando algumas pesquisas sobre educação performativa se aportam exemplos que permitam que os professores enfrentem os desafios que supõe a educação musical no século XXI. Por isso, a metodologia está baseada em uma revisão bibliográfica de fontes primárias e secundárias sobre o que tem sido escrito pelos autores mais influentes no campo da educação performativa. Esta publicação quer enfatizar a importância da pesquisa a nível educativo no âmbito da música, especialmente no ensino instrumental, que na atualidade estão escassos de reflexão. 
Por tanto, o diferencial do texto está nos exemplos que se apresentam como modelos para os professores que queiram implementar uma pesquisa na sua própria aula.

PALAVRAS CHAVE: educação musical performativa - conservatório - didática da música - pesquisa educativa - Ensino musical - classe instrumental - professores.

\section{INTRODUCCIÓN}

El artículo que se presenta aborda un campo poco estudiado de la práctica docente musical. Está basado en el análisis de la praxis pedagógica y educativa de las enseñanzas musicales en su vertiente performativa o práctica. Esta investigación aporta a la comunidad educativa de los conservatorios herramientas para una explicación científica de cómo se enseñan los instrumentos y qué metodologías son las que se llevan a cabo en las aulas.

El objeto de estudio abarca diversos aspectos que no son simplemente la técnica o la metodología utilizada por el profesor, sino algo más trascendente como es la visión global del instrumento en sí. Se trata de elementos más pequeños que deben hacerse medibles, sirvan de ejemplo el vibrato o la técnica de embocadura en los vientos, la colocación o el peso sobre los instrumentos de teclado, ataques y arcos en las cuerdas o el repertorio usado para aprehender la totalidad de concepción del instrumento. Además se investiga cómo se enseñan las características estilísticas y la manera en la que el bagaje del profesor incide en el alumno.

Se trata de aportar un modelo para la investigación docente, en la que los profesores de instrumento expongan su ideario educativo, objetivos y contenidos con los que trabajar, la metodología a utilizar, la evaluación del alumnado y la concreción de todo ello en las unidades didácticas. Es importante destacar que se trata de un modelo aplicable en un contexto general, y que en la práctica debe ser abierto, flexible y adaptable a las circunstancias e individualidades de los diferentes centros y alumnos.

En un momento en el que los centros superiores de música y la comunidad educativa musical apuesta por la investigación, tanto normativamente, como con iniciativas propias como congresos, seminarios... se hace patente esta temática como elemento de discusión indispensable para la educación musical del sigo XXI.

\section{ENSEÑANZA MUSICAL. LA CLASE DE INSTRUMENTO}

La praxis docente no es inmanente en el tiempo, se trata de un devenir cambiante y sujeto a multitud de variables. Esto hace difícil su revisión, que muchas veces se ve relegada a un estudio de la metodología, pero no de la acción docente (Botella y Escorihuela, 2014). En este sentido, la investigación en la clase de instrumento ha de abordar la didáctica instrumental, la producción pedagógica sobre el mismo, las diferentes escuelas y la historia y situación actual de los medios en los que se 
instruye: los conservatorios. Con todo ello, el tipo de análisis que se haga sobre la misma debe crear un marco que contemple todas las variables que intervienen en el aula.

Comúnmente cada profesor de instrumento ha revertido en sus alumnos la técnica o la visión que él ha recibido, pero cuesta encontrar en los conservatorios y centros de educación musical un razonamiento previo, plasmado en una programación didáctica discutida, revisada y adaptada, así como en los proyectos en común dentro de los departamentos. Es decir, se enumeran objetivos, contenidos, incluso resultados de aprendizaje, pero la investigación en el aula supone mucho más.

En España existen una treintena de centros que imparten enseñanza superior de música, entre privados y públicos, número que no para de crecer con nuevas fórmulas en universidades privadas o centros de alto rendimiento exclusivos. Destacan algunos de los más antiguos como el Real Conservatorio Superior de Madrid (1830) o el Conservatorio de Valencia (1879). Así mismo, han alcanzado grandes cuotas de reconocimiento los centros superiores de Aragón, Cataluña o País Vasco, que desde su fundación han apostado por romper con los cánones de los conservatorios clásicos (Escorihuela, 2017).

Las programaciones que cada profesor diseña son un ejercicio de autonomía, tanto de él como del centro. Para entender la didáctica de la clase de instrumento, es preciso saber cómo el profesorado adapta y concreta las intenciones educativas, expresadas en los diferentes elementos del currículo, hasta transformarlas en una propuesta coherente de actividades de aula. El acceso a esta documentación es importante si se quiere abordar un estudio sobre praxis docente, no obstante es posible que esconda prácticas no reconocidas, un escollo que el investigador tiene que superar a través de las distintas técnicas y herramientas de investigación.

Algunos autores han abordado la figura del profesor de instrumento y el análisis de los estudios praxiales de música. Es el caso de Riveiro (2014), que incide en que la formación inicial del profesorado de las enseñanzas instrumentales de música ha de constituir un importante foco de estudio e investigación. La autora demanda una mayor vertiente pedagógica en los niveles superiores de Educación Musical, teniendo en cuenta que la mayoría de los egresados de estos estudios terminan ejerciendo como profesores de música, bien en conservatorios o bien en enseñanzas generalistas. La escasa o inexistente formación didáctica con la que se enfrentan los músicos a la docencia supone un perjuicio a los alumnos.

Elliott (2005) trata la educación práctica que representa la música. Según el autor, una manera de resumir la filosofía práctica es abordar siete cuestiones básicas que para él están presentes en la mayoría de las situaciones de enseñanza-aprendizaje. Éstos ítems a los que se refiere son: los objetivos, los conocimientos, los alumnos, los procesos de enseñanza-aprendizaje, los profesores, el contexto de enseñanzaaprendizaje y la evaluación. La clase de instrumento debe tener como fin la adquisición de la musicalidad. Ésta implica una amplio conocimiento procedimental que se basa en diferentes tipos de saber musical. La musicalidad es sensible al 
contexto, y aunque el conocimiento verbal contribuye de manera importante al desarrollo de la misma, éste es secundario. Así pues, una parte esencial de la tarea del profesor es enseñar a los estudiantes a cómo continuar el desarrollo de su musicalidad en el futuro. Las claves de este proceso se dirigen a la atención musical progresiva, la resolución de problemas, el hallazgo de soluciones, la reducción del problema musical, la reflexión crítica, la generación creativa y la selección de ideas musicales. Todos estos procesos conllevan la participación de los estudiantes en proyectos musicales donde tomen decisiones y se aproximen a prácticas musicales reales. La musicalidad y la capacidad de enseñanza son interdependientes, la una sin la otra son insuficientes. Convertirse en un maestro de música excelente depende mucho de aprender a reflexionar y comprender el esfuerzo de los retos musicales. Por ello, los programas de formación deben preparar a los futuros artistas-docentes a través de unos modelos excelentes de enseñanza y ejemplos de diversos materiales musicales (Elliott, 2005).

Otro autor que aborda la clase de instrumento y la enseñanza práctica musical, es Bowman (2005). Desde la visión práctica de la enseñanza musical, enfatiza la naturaleza de la praxis como acción reflexiva. Es decir, la práctica musical es un hecho consciente, si bien sus implicaciones precisas para aspectos como el contenido curricular o la metodología pueden ser discutibles. El significado de esta convicción fundamental es clara e inequívoca: la Educación Musical orientada a la destreza debe alejarse de las ejecuciones o interpretaciones basadas en la intuición y sin un anclaje a los conocimientos teóricos y estéticos.

Son muchos los autores que han tratado el cambio de paradigma de la clase de instrumento, de una Educación Musical basada en lo práctico a la adquisición de unas competencias que formen a un músico completo. Es el caso de Koopman (2005), para quien las habilidades de hacer música enfocadas exclusivamente en la práctica no se ajustan a la diversidad de papeles musicales que se pueden tomar en la actualidad. El estudiante instrumental debe aprender a interactuar con la música en múltiples situaciones, y someterse a estímulos que lo conviertan en agente musical crítico.

Una de las investigaciones que resulta referencial para los estudios en educación performativa es Performers as teachers: exploring the teaching approaches of instrumental teachers in conservatoires. En ella Purser (2005), encontró muchas similitudes entre profesores de conservatorios de Londres, pero también diferencias sustanciales, debidas al enfoque individual del tipo de enseñanza. Sus resultados plantean la cuestión de proporcionar algún tipo de formación para los profesores instrumentales en el conservatorio. Afirma que los profesores de orquesta en conservatorios, a menudo son nombrados por la importancia de su estatus como intérpretes, primando su valía artística por delante de la pedagógica. Si se tiene en cuenta la forma individualizada de enseñanza que se ha perpetuado se deduce que el aprendizaje ha sido en su mayor parte sin evaluación de la calidad docente.

El análisis de Carruthers (2008) se centra en la música en las escuelas canadienses y el desarrollo de destrezas. La visión generalizada del progreso en la 
enseñanza musical ha radicado en el desarrollo del talento, la aptitud, los aprendizajes previos y la coordinación física. Los nuevos enfoques sobre la enseñanza y el aprendizaje de la música han abierto un abanico de objetivos más amplio. El artículo contrapone ese modelo pedagógico con los resultados esperados de las carreras universitarias en música, especialmente en interpretación. Las enseñanzas basadas en la interpretación todavía pretenden un tipo de aprendizaje que desde hace tiempo es considerado limitante y Restrictivo. Este autor postula un enfoque para la música en el nivel universitario con nuevas metas pedagógicas, que toma como punto de partida los desarrollos innovadores de la Educación Musical escolar.

Una de las conclusiones de Carruthers (2008) es que si los programas performativos de la universidad fueran como los de la escuela -es decir, si cambiasen el énfasis en el producto a el proceso- la relevancia de los músicos profesionales en la sociedad sería mayor. Puesto que la música es un agente del crecimiento personal, el cambio social y la conciencia cultural, su estudio beneficia no sólo a los individuos, sino a la comunidad en su conjunto. Un retorno a los valores musicales básicos no socavará la utilidad de los estudios universitarios en Música.

Las modalidades de aprendizaje de los alumnos de instrumento también son diferentes y se deben de estudiar, tal y como hizo Molumby (2004), basándose en las clases de estudiantes universitarios de flauta travesera. Investigó cómo las actividades basadas en aspectos técnicos y musicales de una selección estándar del repertorio podían ser usadas para que los alumnos expresasen como desarrollaban el proceso de enseñanza-aprendizaje. En este estudio cualitativo de la metacognición, se observó cómo los estudiantes se volvieron más conscientes de su propio estilo de aprendizaje. Mediante la identificación y la utilización de diferentes estrategias de enseñanza se anima a los estudiantes a la comprensión de su potencial dentro de un ambiente de grupo.

Igualmente importante es la necesidad de experiencias de enseñanza temprana en combinación con la adquisición de técnicas pedagógicas durante la Educación Musical, para ello se tiene en cuenta las percepciones de los profesores de instrumento en cuanto a su desarrollo profesional y las técnicas de ensayo de éxito que usan en sus instrucciones (Chaffin, 2009). Todo ello aboca a una revisión de los planes de estudio y a la necesidad de formar a los futuros docentes en competencias pedagógicas. La construcción social de la identidad del músico tiene mucho que ver con un desempeño responsable de su labor, y por tanto se debe de desterrar la dicotomía entre músico intérprete y músico docente. Roberts se postula en la línea de que la preparación docente puede hacer construir la identidad de profesor, pese a las evidencias contrarias en el caso de la Educación Musical (Roberts, 1991).

Las mismas hipótesis lanza Concha (1991), cuando aprecia que centrar la cátedra en el estudio de la música a través del instrumento podría significar replantearse cuestiones básicas de la enseñanza. Así pues, los conocimientos complementarios que emergen de las relaciones entre arte, ciencia y tecnología son inseparables del estudio instrumental y la práctica performativa. Se debe caminar hacia un cambio 
cualitativo programático basado en la transferencia, transformación e integración de estas disciplinas en contenidos curriculares de aprendizaje para los itinerarios de música praxial.

En esta línea de investigación se encuentran Botella y Escorihuela (2014, 2016 y 2017), ahondando en el análisis de la praxis docente y constatando la invisibilidad de lo que ocurre dentro de las aulas de instrumento, un campo abierto a la exploración y sobre el que falta reflexión académica y sistematización.

La idiosincrasia de la clase de instrumento se caracteriza por una gran variedad de praxis docentes que llevan a cabo los pedagogos en cada una de sus especialidades. Es decir, se plantea el problema de la diversidad y heterogeneidad de modelos de aprendizaje que se generan en estas enseñanzas. Esto no es una contrariedad en sí, ya que se trata de una realidad plasmada en diferentes ámbitos y especialidades. El inconveniente emerge cuando se quiere enunciar una práctica común, un modelo (heterogéneo u homogéneo), o una descripción de lo que sucede en las aulas (Escorihuela, 2017).

La figura del profesor de instrumento también debe tenerse en cuenta a la hora de investigar sobre la educación musical performativa. Si se atiende al nivel superior de la Educación Musical, el país asiste a una realidad laboral diferente de sus docentes, en función del cuerpo al que pertenecen y la relación jurídica con la administración pública, o del tipo de contrato en los centros privados o subvencionados. El profesorado también presentará distintos perfiles, más académicos, más solista de instrumento, más camerístico, más orquestal, etc. Además, la naturaleza del centro influye en la libertad de implementar programaciones o programas específicos. Todo ello crea un amalgama desestructurado en la Educación Musical Superior, que queda oculto a la ciencia de la educación y al sistema académico.

Según Cano (1995) en su análisis sobre la situación de los conservatorios en España, "hace aún no demasiado tiempo, los conservatorios considerados importantes en el país contaban entre sus docentes con lo más granada de la profesión" (p. 67). Todos estos profesionales ejercían como docentes a la par que compositores de prestigio, instrumentistas de las mejores orquestas y grupos camerísticos, dando auge y prestigio a los centros en los que impartían clase en todos los niveles. Para la autora, en la actualidad los centros de enseñanza albergan a una mayoría de docentes cuyo único contacto con la música ocurre en las horas de clase.

La autora recapacita en que algo falla en el sistema, inmerso en una especie de endogamia profesional que ocurre en el ámbito de los conservatorios «de tan poco contraste profesional con la vida musical real, con la vida de los conciertos, de la creación o la investigación musicológica» (Cano, 1995, p. 68). A todo esto, añade la desmotivación y falta de estímulo profesional, la masificación y la falta de objetivos claros sobre la función que debe desempeñar el músico en la situación actual. Éstas serían algunas de las razones por las que muchos de los músicos más prestigiosos y 
valiosos del país no quieren ejercer docencia en centros oficiales y prefieren dedicarse a sus carreras performativas tanto en España como en el extranjero. Ante esto, se precisa una reflexión acerca del modelo de Educación Musical que necesita el país. La necesidad o no de flexibilizar los puestos docentes y de modificar los planes de estudios que incluyan formación y práctica pedagógica para un alumno que, en gran medida, va a estar abocado a la docencia aunque su principal faceta sea la interpretativa o performativa. Los futuros grandes intérpretes que egresen del sistema también deben aprender a enseñar, ya que, por su condición, van a ser llamados a ejercer la docencia. Con ello, la investigación en la didáctica de lo performativo es un camino indispensable para el saber científico de la didáctica musical.

\section{INVESTIGACIÓN EN DIDÁCTICA DE LOS INSTRUMENTOS MUSICALES}

La investigación sobre la didáctica de los instrumentos y su praxis docente se ha abordado desde diferentes campos, tanto a nivel de enseñanzas profesionales como amateurs o complementarias. La de la flauta dulce o de pico es la que más ha sido estudiada, ya que resulta una herramienta docente importante en la asignatura de música en la Educación Primaria y la Secundaria. Sin embargo, apenas existen trabajos científicos relacionados con los instrumentos que se estudian de manera profesional en los Conservatorios. Son los instrumentos de cuerda los que más han sido investigados en este campo. Esto se debe a su gran variedad técnica y metodológica, muy arraigada a las escuelas nacionales y que admite una fácil diferenciación y medición de sus resultados. En la mayoría de casos, los docentes tienen claros los objetivos que se pretenden alcanzar y los contenidos a desarrollar, pero muchas veces se deja de lado la reflexión en torno a la práctica en el aula (Botella y Escorihuela, 2017).

En la actualidad existe muy poca bibliografía acerca de la praxis docente instrumental, quizás por su carácter efímero y cambiante; sujeta las particularidades de la ratio $1 / 1$ y condicionada al profesor y centro. Con el actual sistema de cuerpo de profesores puede resultar difícil establecer o sentar lo que se ha venido a llamar escuela o cátedra. Las plantillas son variables por la diversidad de tipología laboral, profesores contratados, interinos, profesores en comisión de servicios, catedráticos... No solo en España pasa esto, se agudiza más en Europa, donde los centros de Educación Musical practican una alta movilidad del profesorado. Todo ello aboca a un campo muy poco estudiado y hace más necesario si cabe abordar este tema que para la comunidad docente puede resultar de gran interés (Escorihuela, 2017).

Por tanto, la investigación en la didáctica de los instrumentos se puede justificar per se, en el sentido de que en sí misma aborda algo de lo que es difícil obtener información. Sirva como ejemplo el estudio de Botella y Escorihuela (2016) sobre la enseñanza de la flauta travesera, en el que afirman: "se sabe poco de lo que el profesorado enseña y cómo lo enseña, de las corrientes flautísticas que se están siguiendo y del tipo de experto de la flauta que una vez acabada su educación superior está saliendo al mercado musical" (p. 412).

Revista de Comunicación de la SEECI. 2021, nº 54, 1-19 
En este sentido, cabe preguntarse qué producto musical está lanzando al mundo profesional el sistema educativo. Por tanto, "se necesita saber qué y cómo se está enseñando para saber en qué y cómo mejorar esta praxis y revisar, incluso exportar, las características propias del tipo de enseñanza" (Botella y Escorihuela 2016, p. 412).

El planteamiento es sencillo en tanto que el problema radica en que existe una realidad no visible de la enseñanza musical interpretativa. Por eso, ante un campo poco estudiado, poco desarrollado y casi inadvertido para los pedagogos e investigadores, se hace preciso resolver en una demostración o evidencia de lo que pasa en las clases. Para así dar testimonio de una manera de hacer, así como de distintos procedimientos para un mismo objetivo.

Investigar sobre la clase instrumental es reflexionar sobre la misma. Metodológicamente, la filosofía de la buena práctica musical parece valorar más la inducción sobre la deducción, la descripción sobre la prescripción, y la evidencia empírica sobre la especulación metafísica. Desde este punto de vista, resulta escéptica la noción de un sustrato fundamental para toda la música (Bowman, 2005). La revisión metodológica y compilación de repertorios, junto a la intervención sobre los sujetos que toman parte del proceso de enseñanza-aprendizaje han sido los métodos más empleados para la investigación en didáctica de los instrumentos musicales.

En este sentido, el enfoque que aporta Purser (2005) resulta del todo adecuado para acercarse a la actitud del profesorado. En su estudio de casos participaron seis artistas de renombre, especialistas en trompeta, trompa, trombón, flauta, clarinete y fagot. Lo que tenían en común es que eran profesores de conservatorios de la misma ciudad y que los estudiantes de estos instrumentos son propensos a centrarse en la práctica orquestal. La investigación se diseñó en dos partes: un cuestionario que pretendía preparar las posteriores entrevistas individuales, y unas entrevistas semiestructuradas. El análisis se llevó a cabo principalmente a través de buscar similitudes y diferencias en las respuestas de los individuos, descubriendo desigualdades según la etapa de desarrollo en su filosofías de enseñanza. Mientras que los profesores con mayor experiencia aportaron más ideas y puntos de vista, los que tenían menos experiencia fueron más limitados en sus respuestas. En líneas generales, este estudio concluye que debe prevalecer lo musical sobre lo técnico, y la formación se debe basar en algo más que una educación en la destreza instrumental. Esta investigación sobre profesores londinenses arrojó algunas conclusiones que pueden servir de punto de partida para otros estudios similares, como que las principales habilidades para alcanzar un alto nivel interpretativo dependen de la capacidad de autocrítica y la importancia de la escucha. Todos los sujetos expresaron su preocupación por la gestión de la relación profesor-alumno. Aunque presentaron algunas estrategias diferentes, la delicadeza y la intimidad que proporciona la ratio $1 / 1$ en la enseñanza era un tema importante para todos. Sin duda alguna, uno de los principios que motivan la investigación en didáctica de los instrumentos es la necesidad de interacción de docentes-artistas en foros para el intercambio de experiencias y modos de buenas prácticas (Purser, 2005). 
Muchas de las investigaciones sobre didáctica en el aula han usado como técnica de investigación la encuesta. Ésta sirvió también a Botella y Escorihuela (2017) para conocer la actitud del profesorado español de flauta travesera de las enseñanzas superiores de música, mediante un cuestionario que les permitió expresar su parecer acerca de la mejor manera de enseñar este instrumento y cuáles son los pilares en los que fundamentan la formación que dan a los alumnos. El instrumento utilizado en este caso fue un cuestionario mediante el cual los profesores aportaron información sobre sus prácticas docentes. Se trata de un ejemplo de investigación en educación performativa reciente, mediante una técnica que ha proporcionado resultados válidos y que puede generalizarse, por ello este artículo toma este ejemplo para presentar un modelo universal.

\section{LA METODOLOGÍA DE COMPLEMENTACIÓN COMO PROPUESTA PARA LA INVESTIGACIÓN EN DIDÁCTICA DE LOS INSTRUMENTOS MUSICALES}

La enseñanza de lo efímero en la música, así como las técnicas de lo perdurable en la misma, necesitan de una metodología de investigación que combine aspectos cualitativos y cuantitativos. En la investigación educativa se hace necesario recurrir a técnicas evaluativas de diferente índole que deben complementarse, de esta manera es como se produce un análisis integrado (Moscoloni, 2005).

Las enseñanzas musicales pueden beneficiarse así de los avances logrados en los últimos años gracias a planteamientos cualitativos en disciplinas como Didáctica General y otras Didácticas Específicas. En este sentido, se puede decir que la investigación cualitativa intenta desvelar los significados que los humanos le dan a las acciones que desarrollan, con una finalidad no solamente descriptiva sino con la intención de proponer alternativas para su mejora, mientras que la investigación cuantitativa, aporta los datos empíricos y comprobables métricamente, que permiten entender y explicar los diferentes comportamientos en la acción docente (RodríguezQuiles, 2000).

A través de la investigación de Escorihuela (2017) sobre la enseñanza de la flauta travesera, se observa un estudio sobre la praxis docente basado en la complementación de distintas metodologías. En este caso, las variables cuantitativas se discuten a partir de un análisis métrico, mientras que el análisis de las variables cualitativas se realiza mediante la construcción conceptual de categorías a las que cada profesor se adscribe. Además, se añade un análisis relacional entre variables, que mide el grado de asociación entre las mismas.

La investigación educativa, y más en concreto la perfomativa musical, necesita trabajar con los sujetos del proceso de enseñanza-aprendizaje. Las características eminentemente prácticas e individualizadoras de estos estudios así lo exigen. Desde este punto de vista cobra especial importancia el docente, no sólo como testigo directo de la realidad que se pretende estudiar, sino como eje vertebrador de una manera de interpretar, de hacer música y músicos. Por eso interesa por igual la práctica docente, la programación y el perfil del profesor. 
Tal y como apunta Rodríguez-Quiles (2000), la educación es un proceso esencialmente humano que no puede escapar a la Ciencia, de esta manera surgen los primeros trabajos etnográficos a finales de los años setenta en Gran Bretaña, Estados Unidos y Australia. Por ello plantea que, en consecuencia, "la Educación Musical, como parte de una formación integral del individuo, podrá verse beneficiada por una investigación de corte cualitativo correctamente planteada" (pp. 2-3).

Según Díaz y Giráldez (2013), la etnografía educativa contribuye a descubrir la complejidad de los fenómenos educativos, además aporta un conocimiento real y profundo de los mismos. Es decir, supone un complemento a la investigación, que se ve reforzada y es capaz de contribuir en mayor medida al conocimiento de la comunidad científica.

Escorihuela (2017) se hace valer de un estudio de casos desde una perspectiva etnográfica para su análisis de la enseñanza del instrumento. Por sus características, el estudio de casos es difícil de estructurar con unos pasos delimitados (Stake, 1998), por ello el investigador debe definir muy bien sus objetivos y adecuar el estudio de casos. En el de Escorihuela (2017) se describen los sistemas educativos de tres países que son referencia en Educación Musical, para poder hacer una comparación con el sistema español. La formulación de la investigación se hace a partir de observaciones y reflexiones de los datos que se obtienen. Se trata de un método que permite adaptarse al contexto.

Si bien las técnicas exploratorias cuantitativas poseen gran adecuación para el tratamiento de datos sociales y propios de la educación, Moscoloni indica que "la determinación a priori de las categorías de los indicadores puede producir a veces interpretaciones algo superficiales" (2005, p. 9).

La caracterización cualitativa de los docentes es muy importante en este tipo de investigaciones, que buscan un prototipo $o$ un modelo. Por tanto, hay que delimitar las variables que van a explicar una determinada praxis, y que son generadoras de estandarización. Una de las variables más aclaratorias en educación musical para instrumentos es la que hace referencia al uso de libros de estudio y técnica, lo que se han venido a denominar métodos.

Además del método o métodos, la enseñanza de un instrumento también puede verse influenciada por la escuela. Este concepto abarca la metodología empleada por el maestro o profesor, o el conjunto de docentes de una institución. En el ámbito del arte, la escuela define al grupo formado por los seguidores, admiradores o aprendices de un individuo que actúa como maestro o fuente de inspiración. Las escuelas nacionales de cada instrumento han jugado un papel importante en la generalización y el exponencial de las técnicas y los métodos que han conseguido ser atemporales y de probada solvencia (Botella y Escorihuela, 2017).

Con todo ello, las variables que se tengan en cuenta a la hora de investigar la praxis docente no serán siempre estables, pues en cada lugar se aprende la técnica 
instrumental con unas características propias, normalmente resultado de la influencia de uno o más conocidos instrumentistas de la zona en cuestión. En el caso de la escuela, los viajes, las grabaciones y la radio están borrando poco a poco las diferencias. La escuela como concepto de ejecución basado en la enorme influencia de un gran flautista a través de su método pedagógico, sus libros y su manera de tocar queda en entredicho (Botella y Escorihuela, 2016).

Así pues, la selección de casos o la de una muestra de la población de estudio, puede presentarse como una opción adecuada para complementar los análisis cuantitativos y cualitativos. Todo dependerá de los objetivos de la investigación y del ámbito de la misma. Un trabajo que pretenda reflejar la práctica docente de una determinada etapa educativa, deberá abordar cuestiones que hagan referencia a los propios docentes: lo que pretenden conseguir con su alumnado y la metodología que utilizan. Para conocer la praxis docente de los profesores de las enseñanzas instrumentales es preciso deshilar este objetivo marco, ya que la didáctica performativa supone un entramado entre el bagaje del profesor, la escuela, la técnica, los métodos, las obras del repertorio, aspectos psicológicos, etc. Para llevar a cabo este cometido se deben plantear objetivos específicos que se relacionen directamente con la estructura de cada investigación

Sirva como ejemplo el caso que nos muestran Botella y Escorihuela (2016), donde se puede observar que los objetivos engloban: describir la práctica didáctica de los Conservatorios Superiores de la Comunidad Valenciana, observar y determinar a qué perfil flautístico se adscribe cada profesor y cómo influye en el tipo de alumnado, conocer los autores de referencia que se interpretan tanto en el repertorio como en los libros de técnica y de estudios, y si los profesores siguen un programa común de centro o cada uno prepara su programación, así como descubrir en qué grado el bagaje musical de cada profesor influye en su manera de impartir clase de flauta y de programar.

Todos estos objetivos se deben plasmar en las variables, que deben hacerse ponderables mediante las mediciones empíricas de los datos cuantitativos. Éstos proporcionan una visión de la realidad a través de a cual se puede elaborar un modelo conceptual que describa e interprete esta realidad, apoyándose en datos cualitativos.

La herramienta que aglutina ambas metodologías, y se ha demostrado como solución para la complementación, es el cuestionario. Instrumento mediante el cual el investigador puede acercarse al objeto de estudio a través de preguntas cerradas de corte cuantitativo, y otras abiertas, en las que el encuestado puede expresarse libremente y aportar el valor cualitativo.

\section{LA TÉCNICA DE CUESTIONARIO: UNA HERRAMIENTA PROBADA PARA TESTAR LA PRAXIS DOCENTE}

Los conservatorios son el medio en el que se desarrolla mayoritariamente la actividad docente musical de carácter práctico. Antes del nacimiento generalizado de 
estos centros en España hubo otros medios para formarse musicalmente, la mayoría de carácter colectivo: ingresar como aprendiz en un grupo instrumental o en las bandas militares. Sin embargo, la clase particular fue el medio individual más común para la enseñanza del solfeo y de los instrumentos.

Con estos antecedentes y el espíritu decimonónico con el que se fundan los conservatorios, no es de extrañar que sus comunidades educativas se refugien en un institucionalismo reservado y poco dado a la innovación educativa, la investigación y la transferencia. Con metodologías que se mantienen sin muchos cambios desde hace más de cien años, a ello ayuda el formato que se ha mantenido de clase magistral y ratios a uno, dando lugar a fuertes individualidades.

Por todo ello, se considera que el cuestionario es la mejor manera de entrar masivamente al conocimiento de la praxis de los docentes instrumentales, en parte por su anonimato, que preserva las personalidades y la intimidad de la clase individual y, por su sistematización y el uso de unidades de medida estándares ${ }^{3}$.

Tal y como indica Johansson (2011) en un artículo que se refiere a la enseñanza vocal e instrumental uno a uno en conservatorios, la tarea de la capacitación profesional en estos entornos ha sido lograr un nivel de experto, que a menudo se describe como una etapa que se alcanza paso por paso por progresión. El trabajo se realizó sobre la población de un solo centro, por lo que la técnica empleada fueron los grupos focales además de la entrevista y seguimiento del personal. En este caso, y para contestar a las preguntas: ¿Cuáles son los objetivos de los docentes en la enseñanza y cómo lo hacen? ¿Cómo definen el desarrollo?, las entrevistas cualitativas fueron la mejor herramienta para captar el conocimiento incorporado en el tradición del conservatorio.

Si se quiere tener un conocimiento certero de distintos centros y abarcar una gran población, el cuestionario se revela como la técnica más adecuada. Éste tiene que tener en cuenta el tipo de enseñanza musical profesionalizadora que se da en los centros de Educación Musical. El objetivo primordial de éstos está especialmente orientado a promover músicos de gran talento de forma selectiva y potencialmente para prepararlos hacia una carrera musical (Altenburg, Bässler y Nimczik, 2011).

Un cuestionario debe combinar preguntas abiertas y cerradas. Las primeras dan cuenta de la investigación cualitativa mientras que las segundas se hacen de eco de la parte cuantitativa. Estos estudios utilizan el método encuesta para determinar la actitud de los profesores a la hora de dar clase a sus alumnos. Con él se obtiene

\footnotetext{
${ }^{3}$ Por supuesto que la entrevista es otra de las herramientas utilizables para conocer la praxis de los profesores de instrumento, tal y como reflejan multitud de estudios. Sin embargo, la misma excluye datos cuantitativos que pueden ser explicativos de la docencia y la manera de hacer de una determinada población.
} 
importante información, ya que se trata de la fuente primaria más relevante que puede aportar las respuestas sobre la enseñanza actual de un instrumento musical.

Los trabajos de Botella y Escorihuela (2016 y 2017) se centran en un estudio vinculado a la praxis de la flauta en las aulas de los centros superiores de música, que sirve para conocer las prácticas docentes del profesorado, mediante un cuestionario que les permita expresar su parecer acerca de la mejor manera de enseñar este instrumento y cuáles son los pilares en los que fundamentan la formación que dan a los alumnos. Las técnicas de muestreo que se utilizan en investigación permiten determinar la parte de la realidad estudiada (población) que debe examinarse con objeto de hacer inferencias sobre dicha población. Los autores tratan de obtener una muestra adecuada que permita lograr una versión simplificada de la población y que reproduzca sus rasgos básicos con la precisión fijada (Botella y Escorihuela, 2017). Los criterios básicos que se deben tener en cuenta en la elaboración de un cuestionario son la sencillez, precisión y concreción de los ítems que los compone, así como la discreción y preservación del anonimato en el manejo de la información recabada. Botella y Escorihuela (2017) se decantan por este procedimiento de recogida de información altamente estructurado, en el que plantean una serie de preguntas en torno a las variables que se quieren medir. Con ello consiguieron reunir mucha información en relativamente poco tiempo.

El investigador que se centre en la praxis de la docencia musical y opte por esta técnica, tendrá que diseñar un cuestionario a partir de una revisión bibliográfica extensa, sobre la técnica instrumental, su estudio, métodos y evolución. Es recomendable una primera revisión hecha por expertos del ámbito universitario especializados en evaluación de programas, que observen la adecuación del vocabulario, redacción y el uso correcto de parámetros de medida.

Siguiendo el criterio de triangulación, una segunda revisión debe ser llevada a cabo por contraste de expertos de validación de contenido que verifiquen que los ítems propuestos miden la didáctica instrumental. En este sentido, la validez de contenido es el grado en que una prueba representa de forma adecuada lo que se ha realizado.

Así pues, en la elaboración del cuestionario se debe tener en cuenta la definición del constructo a medir, el propósito de la escala que se use, la composición de los ítems determinada por el número, contenido, la definición y ordenación, y la prevención de los sesgos en su cumplimentación y codificación de las respuestas.

Es importante seleccionar unas variables de información y clasificación en cada estudio que permitan la estructuración del cuestionario configurando los distintos ítems. En el caso de la investigación de Botella y Escorihuela (2016), la estructura del cuestionario se compone de un cuadro inicial que contiene las variables de clasificación para caracterizar la muestra. Les siguen 5 dimensiones relativas al perfil del profesor, colocación y embocadura, estudio del sonido, digitación y la articulación, estudio y repertorio. 
Respecto a la tipología y caracterización de las preguntas, un cuestionario que investigue la educación performativa musical debe contener preguntas cerradas, semiabiertas y abiertas. Las preguntas cerradas pueden generar distintas posibilidades de respuestas: excluyentes (respuestas dicotómicas) o con varias opciones de respuesta. Para este tipo de estudios se recomiendan las dicotómicas porque requieren de un menor esfuerzo por parte de los encuestados, son sencillas de contestar y mantienen atento al sujeto en el tema. También se sugieren las escalas de tipo Likert, pues a través de ellas se consigue la reacción favorable o desfavorable, positiva o negativa, y son de fácil medición. Las preguntas semiabiertas puede derivarse directamente de las anteriores, cuando se contempla la posibilidad de añadir alternativas no incluidas en el cuestionario para enriquecer el análisis. Con las preguntas abiertas, los encuestados pueden opinar libremente sobre lo que se les demanda e incluir la información que deseen sobre sus situaciones personales. Son la mayor fuente de datos cualitativos, y además permiten matizar las respuestas cerradas y añadir nuevas perspectivas sobre el tema tratado.

Tal y como afirman Botella y Escorihuela (2016): "una vez diseñado el borrador definitivo, delimitada la información, formuladas las preguntas, definido el número de ellas y ordenadas, hay que realizar una evaluación de las propiedades métricas de la escala" (p. 412).

En todo caso, el cuestionario debe ser sometido a validez de contenido, constructo y criterio. En el primer caso se corrobora que los ítems elegidos son indicadores de lo que se pretende medir, exponiendo el documento a la valoración de investigadores y expertos, que deben juzgar la capacidad de éste para evaluar todas las dimensiones que se desea mesurar. La segunda de las valideces evalúa el grado en que el instrumento refleja la teoría del fenómeno o del concepto que mide, garantizando que las medidas que resultan de las respuestas del cuestionario pueden ser consideradas y utilizadas como medición del fenómeno. Puede ser calculada por diversos métodos, pero los más frecuentes son la matriz multirasgo-multimétodo y el análisis factorial. Por último, la validez de criterio hace referencia a la relación de la puntuación de cada sujeto con un Gold Standard que tenga garantías de medir lo que se desea. Cuando no hay indicadores de referencia se recurre a utilizar herramientas que han sido respaldadas por otros estudios.

Con todo ello, el cuestionario es el documento que más información proporciona, dando validez al estudio y pudiendo servir de modelo para la investigación de cualquier enseñanza performativa musical. Botella y Escorihuela (2016) valoran positivamente este método, ya que es en el que más profesores se implicaron. Para algunos sujetos la entrevista puede introducir comentarios y apreciaciones, difíciles de explicar con el método encuesta. No obstante, la justificación de esta decisión viene motivada por los objetivos de la investigación, en este caso se trata de obtener datos a nivel nacional $y$, por eso, es mejor el procedimiento de cuestionario. Se asume el sesgo de información, que es mucho más entendible en el cara a cara, pero se gana en resultados estadísticos y un prototipo o modelo de enseñanza del instrumento en el país. 


\section{CONCLUSIONES}

La educación performativa musical debe abordar el pleno dominio de los problemas de ejecución que plantea el repertorio del instrumento, una tarea prioritaria para el intérprete que absorbe un tiempo considerable dentro del total de horas dedicadas a su formación musical global. Una investigación sobre la misma, ha de tener muy en cuenta que el trabajo técnico debe estar siempre indisociablemente unido en la mente del intérprete a la realidad musical a la que se trata de dar cauce.

La investigación sobre la praxis docente musical se abre paso ante un panorama en el que los Estudios Superiores de Música se imbrican en el Espacio Europeo de Educación Superior. En este terreno surge la necesidad y la perentoriedad de investigar sobre música. Tal y como indica Pastor (2012), las enseñanzas musicales deben subirse al carro de la investigación y lo que propone este artículo es que esta investigación se haga también desde la vertiente pedagógica, no sólo histórica, performativa o tecnológica. De alguna manera se reivindica la investigación en la educación praxial de la música y se dota a los docentes-investigadores de herramientas en base a ejemplos ya realizados en el campo.

El desafío que supone incorporar y mantener la tradición, y desarrollar una musicalidad independiente y expansiva, es una búsqueda inherente en los buenos docentes de música. Esta búsqueda debe realizarse a través de la investigación, con las herramientas adecuadas que permitan al docente-investigador mejorar su práctica e implementar modelos que sirvan de ejemplo a toda la comunidad científica.

Este artículo ha demostrado, a través de diferentes estudios las ventajas del cuestionario como herramienta para la investigación en educación musical performativa. La propuesta se puede aplicar a multitud de estudios sobre la enseñanza instrumental, ya que emplea un modelo basado en la clase tradicional que nace del Conservatorio de París en el siglo XIX y evoluciona a hoy en día, con el constructivismo de base. Este molde que divide la clase en secciones también sirve para distribuir el cuestionario en dimensiones. Compaginando cuestiones cuantitativas con cualitativas, se ha podido evidenciar que la mejor manera de acercarse a la realidad de la didáctica de los instrumentos musicales es usando una metodología de complementación.

En definitiva, todo lo práctico y su didáctica es difícil de mesurar en los parámetros habituales de las ciencias sociales, por ello la enseñanza de lo cambiante trata de hacer medible el arte del momento. Las investigaciones que se realicen sobre estas disciplinas tendrán que buscar las herramientas y tener en cuenta la técnica de lo efímero y la técnica de lo perdurable, por lo que suponen un reto para la educación musical en el siglo XXI. 
Botella, A. M. y Escorihuela, G. Educación performativa musical: herramientas para la investigación

\section{REFERENCIAS}

Altenburg, D., Bässler, H, y , Nimczik, O. (2011). Education for Musical Professions. En Schulmeistrat, S. y Wallscheid, M (Eds.) Musical Life in Germany: Structure, facts and figures (pp. 68-92). Bonn: German Music Council.

Botella, A. M. y Escorihuela, G. (2014). Análisis de la praxis docente de los profesores de las enseñanzas superiores de flauta travesera de la Comunidad Valenciana. El Artista, 11, 65-87.

Botella, A. M. y Escorihuela, G. (2016). Educación performativa: La enseñanza superior de la flauta travesera en la Comunidad Valenciana. En Actas del II Congreso Nacional de Conservatorios Superiores de Música (pp. 410-425). Madrid: SEM-EE.

Botella, A. M. y Escorihuela, G. (2017). Diseño y validación de un cuestionario para conocer la praxis docente del profesorado de flauta travesera en centros superiores de la Comunidad Valenciana. Revista de Comunicación de la SEECI, 42, 1-13. https://doi.org/10.15198/seeci.2017.42.1-13

Bowman, W. D. (2005). The Limits and Grounds of Musical Praxialis. En Elliott, D.J. (Ed.) Praxial Music Education. Reflections and Dialogues (pp. 3-18). New York: Oxford University Press.

Cano, A. (1995). Aprender música en el conservatorio. Eufonía, 1, 65-72. https://hdl.handle.net/11162/197985

Carruthers, G. (2008). Educating professional musicians: lessons learned from school music. International Journal of Music Education, 26, 127-135. https://doi.org/10.1177/0255761407088487

Chaffin, C. (2009). Perceptions of instrumental music teachers regarding the development of effective rehearsal techniques. Bulletin of the Council for Research in Music Education, 181, 21-36.

Díaz, M. y Giráldez, A. (2013). Investigación cualitativa en educación musical. Barcelona: Graó.

Elliott, D. J. (2005). Praxial Music Education. Reflections and Dialogues. New York: Oxford University Press.

Escorihuela, G. (2017). La enseñanza de la flauta travesera en los centros superiores de música de España (Tesis doctoral, Universitat de València). Recuperado de http://roderic.uv.es/handle/10550/62948 
Botella, A. M. y Escorihuela, G. Educación performativa musical: herramientas para la investigación

Johansson, K. (2012). Experts, entrepreneurs and competence nomads: the skills paradox in higher music education. Music Education Research, 14, 45-62. https://doi.org/10.1080/14613808.2012.657167

Koopman, C. (2005). The nature of music and musical Works. En Elliott, D.J. (Ed.) Praxial Music Education. Reflections and Dialogues (pp. 79-97). New York: Oxford University Press.

Molumby, N. (2004). The application of different teaching strategies reflective of individual students' learning modalities in the University Flute Studio (Tesis doctoral, Ohio State University). Recuperado de http://rave.ohiolink.edu/etdc/view?acc num $=0$ su1086122579

Moscoloni, N. (2005). Complementación metodológica para el análisis de datos cuantitativos y cualitativos en evaluación educativa. Revista Electrónica de Metodología Aplicada, 10, 1-10. https://doi.org/10.17811/rema.10.2.2005.1-10

Pastor, V. (2014). El proceso de adaptación de los Estudios Superiores de Música en España al Espacio Europeo de Educación Superior. ArtsEduca, 7, 44-64.

Purser, D. (2005). Performers as teachers: exploring the teaching approaches of instrumental teachers in conservatoires. British Journal of Music Education, 22, 287-298. https://doi.org/10.1017/S0265051705006546

Riveiro, L. (2014). El conocimiento pedagógico y didáctico del profesorado de las enseñanzas artísticas musicales. Dedica. Revista de Educação e Humanidades, 6, 295-308. https://doi.org/10.30827/dreh.v0i6.6981

Roberts, B. (1991). Music teacher education as identity construction. International Journal of Music Education, 18, 30-39.

Rodríguez-Quiles, J. A. (2000). Investigación Cualitativa en Educación Musical: un nuevo reto en el contexto educativo español. Revista LEEME, 5, 1-6.

Stake, R. (1998). Investigación con estudio de casos. Madrid: Ediciones Morata.

Zaldívar, Á. (2005). Las enseñanzas musicales y el nuevo Espacio Europeo de Educación Superior: El reto de un marco organizativo adecuado y la necesidad de la investigación creativa y «performativa». Revista Interuniversitaria de Formación del Profesorado, 19, 95-122.

\section{AUTORES:}

\section{Ana María Botella Nicolás}

Doctora en pedagogía por la Universitat de València. Es Licenciada en Geografía e Historia, especialidad Musicología y maestra en Educación Musical, por la Universidad 
de Oviedo. Grado profesional en la especialidad de piano. Durante el año 2001 obtiene por oposición plaza en el cuerpo de profesores de música de enseñanza secundaria en Alicante (actualmente en excedencia). Ha presentado diversas comunicaciones en jornadas y congresos sobre didáctica de la música así como distintas publicaciones. Es profesora contratada doctora del departamento de didáctica de la Expresión Musical, Plástica y Corporal de la Facultad de Magisterio de la Universitat de València. Forma parte de la Comisión de Coordinación Académica del Master Universitario en Profesor/a de enseñanza secundaria de la UVEG y del Máster de Investigación en didácticas específicas.

Orcid ID: https://orcid.org/0000-0001-5324-7152

ResearchID: N-1375-2014

Google Schoolar: https://scholar.google.es/citations?user=AEq28xAAAAAJ\&hl=es https://uv.academia.edu/ABotellaNicolás

\section{Guillem Escorihuela Carbonell:}

Doctor en didáctica de la música por la Universitat de València. Es titulado Superior en Música en la especialidad de Flauta Travesera por el Conservatorio Superior de València. Máster en Profesor de Educación Secundaria y Máster de Investigación en Didácticas Específicas por la Universitat de València. Máster Universitario en Interpretación Musical por la Universidad Internacional de Andalucía. Desde 2015 es profesor del Departamento de Orquesta y Música de Cámara del Conservatorio Superior de Música de Castellón, donde se encarga de la coordinación de Actividades Culturales del centro.

Orcid ID: https://orcid.org/0000-0001-5735-3841 https://conservatorisuperiorcastello.academia.edu/GuillemEscorihuela 